\title{
Highly Unsaturated Fatty Acid might Act as an Antioxidant in Emulsion System Oxidized by Azo Compound
}

\author{
Naohiro Gotoh ${ }^{1}$, Yosuke Noguchi ${ }^{1}$, Akiko Ishihara ${ }^{1}$, Kaita Yamaguchi ${ }^{1}$, Hoyo Mizobe ${ }^{2}$, \\ Toshiharu Nagai ${ }^{2}$, Ikuko Otake ${ }^{2}$, Kenji Ichioka ${ }^{2}$ and Shun Wada ${ }^{1 *}$ \\ ${ }^{1}$ Department of Food Science and Technology, Tokyo University of Marine Science and Technology (4-5-7 Konan, Minato-ku, Tokyo 108-8477, \\ JAPAN) \\ ${ }^{2}$ Tsukishima Foods Industry Co. Ltd. (3-17-9 Higashi Kasai, Edogawa-ku, Tokyo 134-8520, JAPAN)
}

\begin{abstract}
Now it is recognized that DHA is oxidatively stable fatty acid compared with linoleic acid (LA) in emulsified system, although DHA is oxidatively unstable in a bulk system. In fact, an emulsified mixture of DHA and LA behaves as in a bulk system, namely the oxidative stability of DHA becomes lower than that of LA. Therefore, in this study, tridocosahexaenoate (DDD) and glycerol trilinoleate (LLL) were separately emulsified using TritonX-100 as an emulsifier and DDD emulsion was mixed with the oxidizing LLL emulsion using a water-soluble radical initiator, 2,2'-azobis(2-aminopropane) dihydrochloride. As a result, DHA suppressed the oxidation of LA, while DHA was not significantly oxidized. This suppression ability was examined using glycerol trieicosapentaenoate, glycerol trilinolenate, or glycerol trioleate instead of DDD and it was found that this activity was increased with the increasing number of double bonds in the structure. Furthermore, the same type of experiment was carried out using a lipid-soluble radical initiator, 2,2'-azobisisobutyronitrile and the similar result was obtained. These results indicated that a highly polyunsaturated fatty acid might act as an antioxidant in an emulsion system oxidized by an azo compound.
\end{abstract}

Key words: antioxidant, DHA, emulsion, HUFA, linoleic acid

\section{INTRODUCTION}

The oxidation of fats and oils is a significant concern for the food and feed industry because oxidized fats and oils in foods emits a rancid smell ${ }^{1}$, causes food toxicity ${ }^{2-4)}$ in extreme cases, and becomes a fire hazard ${ }^{5)}$. For example, Japan had a food poisoning epidemic caused by the degradation of fats and oils in instant noodles about 40 years ago ${ }^{6)}$. After the incidences, that oxidized fats and oils develop strong neural toxicity was reported. Thus, it was reported that oxidized fats and oils develop strong neural toxicities $^{7)}$. Fish oil is one of the most easily oxidizable oils in bulk system and the oxidation sometimes accompanies heat generation. Fishmeal, containing about $10 \%$ fish oil $^{8)}$, is used for livestock and cultured fish feed and required special attention, particularly when transported across the equator by container ships because the heated containers accelerate the oxidation of fish oil in fish meal. This strong oxidation can cause shipboard fires; therefore, fishmeal is normally sprayed with an antioxidant such as ethoxyquin prior to shipping to prevent oxidation ${ }^{9)}$. The oxidation of fats and oils is essentially the oxidation of polyunsaturated fatty acids (PUFA). PUFA structures contain two or more double bonds, normally located on both sides of a methylene group; in other words, the methylene group is packed by two double bonds. The hydrogen in the methylene group reacts easily with active oxygens or oxygen species and this reaction initiates the oxidation of $\mathrm{PUFA}^{10)}$. The number of methylene groups packed by double bonds in-

Abbreviations: AAPH, 2,2'-azobis(2-aminopropane) dihydrochloride; AIBN, 2,2'-azobisisobutyronitrile; $\beta$-LDL, 1,3-dilinoleate-2-docosahexaenoate; DDD, glycerol tridocosahexaenoate; EEE, glycerol trieicosapentaenoate; HUFA, highly unsaturated fatty acid; LA, linoleic acid; LLL, glycerol trilinoleate; LnLnLn, glycerol trilinolenate; OOO, glycerol trioleate; PUFA, polyunsaturated fatty acid; TAG, triacylglycerol \footnotetext{
4-5-7 Konan, Minato-ku, Tokyo 108-8477, JAPAN

E-mail: wada@kaiyodai.ac.jp

Accepted June 10, 2010 (received for review May 12, 2010)

Journal of Oleo Science ISSN 1345-8957 print / ISSN 1347-3352 online

http://www.jstage.jst.go.jp/browse/jos/
}

*Correspondence to: Shun Wada, Department of Food Science and Technology, Tokyo University of Marine Science and Technology, 
creases with the number of double bonds existing in the fatty acid. For example, $\alpha$-linolenic acid (C18:3n-3) and arachidonic acid (C20:4n-6) possess two and three methylene groups packed by double bonds, respectively. Accordingly, PUFA consisting of many double bonds, such as EPA (C20:5n-3) and DHA (C22:6n-3), characteristically contained in fish oil, are sometimes called highly unsaturated fatty acid (HUFA), and are recognized as easily-oxidizable fatty acids ${ }^{9)}$. However, this is only true in bulk systems because the order of oxidation for the fatty acids is reversed in an emulsified system. For example, Bruna et al. demonstrated that oxidation rates of emulsified EPA and DHA were substantially lower than that of linoleic acid (LA, C18:2n-6) in a photoxidation system ${ }^{11)}$. Miyashita et al. also reported a similar tendency using PUFA and HUFA in $\mathrm{Fe}^{2+}$ and ascorbic acid oxidation system ${ }^{12)}$. They showed that the oxidative stability of micelle consisting of DHA and LA mixture decreased according to the increase in the LA ratio. Interestingly, the oxidation speed of DHA was higher than that of LA in the mixture of DHA and LA micelle. Furthermore, Miyashita et al. indicated that fish oil fatty acid micelle ${ }^{13)}$ and emulsified fish oil ${ }^{14)}$ also possesses the same oxidative stability. Yazu et al. confirmed this characteristic in HUFA using a micelle system oxidized by a water-soluble azo compound ${ }^{15,16)}$. Nowadays, in Japan, Tofu containing fish oil is patented ${ }^{17)}$ and is thus in the market using this knowledge.

Though many researchers have confirmed the oxidative stability of emulsified HUFA, the mechanism is still unclear. Furthermore, as mentioned above, oxidative stability of each fatty acid in micelle of DHA and LA mixture is understood to behave like that of DHA, i.e., with a lower oxidative stability than $\mathrm{LA}^{12)}$. However, another interpretation is that DHA decreases the content because of antioxidant action against LA oxidation in the system. To clarify these points, we separately emulsified HUFA and LA, and their mixture was oxidized using an azo compound in this study.

\section{EXPERIMENTAL PROCEDURES}

\subsection{Chemicals and materials}

Triacylglycerols (TAGs) such as glycerol tridocosahexaenoate (tri-DHA, DDD), glycerol trieicosapentaenoate (triEPA, EEE), glycerol trilinolenate (tri- $\alpha$-Ln, LnLnLn), glycerol trilinoleate (LLL), glycerol trioleate (OOO), and glycerol 1,3-dilinoleate-2-docosahexaenoate ( $\beta$-LDL) were obtained from Tsukishima Foods Industry Co., Ltd. (Tokyo, Japan). The purities of TAG samples were more than $99 \%$. Fatty acid methyl ester standard was bought from SigmaAldrich Japan K.K. (Tokyo, Japan). Other reagents were purchased from Wako Pure Chemical Industries, Ltd. (Osaka, Japan).

\subsection{Preparation of emulsion samples}

TAG was mixed with tridecanoic acid methyl ester (C13) as an internal standard in $50 \mathrm{mM}$ phosphate buffer ( $\mathrm{pH} 7.4$ ) containing $0.5 \%$ TritonX-100. The mixture was emulsified using an ultrasonic homogenizer (SONIFIER S-250, BRANSON Ultrasonic Corporation, CT, USA ${ }^{18)}$. The final concentrations of emulsified TAG and C13 were adjusted to $5 \mathrm{mM}$ and $10 \mathrm{mM}$, respectively.

\subsection{Oxidation of emulsified TAG with 2,2'-azobis} (2-aminopropane) dihydrochloride (AAPH) and measurement of remaining fatty acid with GC-FID

Emulsified TAG or a blend of two types of emulsified TAGs was mixed with AAPH (final concentration was 1 $\mathrm{mM}$ ) aqueous solution. The emulsion was aliquated to fifteen screw-cap test tubes. The test tubes were covered with aluminum foil and oxidized in a water bath at $25^{\circ} \mathrm{C}$ for $96 \mathrm{~h}$. Three test tubes were sampled at 0, 24, 48, 72, and $96 \mathrm{~h}$ after the oxidation started. To each sample test tube, four times the sample volume of an ethanol and isopropyl ether mixture $(1: 3, \mathrm{v} / \mathrm{v})$ was added and the test tubes were centrifuged at $700 \times \mathrm{g}$ for $5 \mathrm{~min}$ under room temperature to extract the TAG. The upper layer of the centrifuged test tube, namely the ether layer, was collected and dried with a rotary evaporator. The residue was methyl esterified using boron trifluoride methanol solution to devote to gas chromatography-flame ionization detection (GC-FID) ${ }^{18)}$. The methyl esterified fatty acid was extracted with hexane and the hexane solution was subjected to a GC-FID system (GC14B, Shimadzu Corporation, Kyoto, Japan) equipped with a capillary column (Omegawax320, $30 \mathrm{~m} \times 0.25 \mathrm{~mm}$ ID, Sigma-Aldrich Japan K.K.) and a Chromatopac integrator (C-R6A; Shimadzu) to analyze the fatty acid composition and relative ratio. The temperatures of the injection port and detector were $250^{\circ} \mathrm{C}$ and $260^{\circ} \mathrm{C}$, respectively. The initial column temperature of $175^{\circ} \mathrm{C}$ was increased to $225^{\circ} \mathrm{C}$ at the rate of $1^{\circ} \mathrm{C} / \mathrm{min}$. Helium was used as the carrier gas at a flow rate of $32 \mathrm{~cm} / \mathrm{s}$. The fatty acid species was identified using the retention time of a fatty acid methyl ester standard solution (Supelco 37 Component FAME Mix, Sigma-Aldrich Japan K. K.). The content of the respective fatty acids was expressed as a relative existence ratio and calculated using a GC-FID chromatogram. The ratio of chromatogram peak area for the fatty acids constructing TAG and $\mathrm{C} 13$ at 0 hour was set as 100; the relative existence ratio of the fatty acids at each sampling time was calculated by the equation([Peak Area of Objective Fatty Acid at Sampling Time]/[Peak Area of C13 at Sampling Time $]) /([$ Peak Area of Objective Fatty Acid at 0 hour $] /$ [Peak Area of $\mathrm{C} 13$ at 0 hour] $) \times 100$.

2.4 Addition of emulsified TAG into LLL emulsion previously oxidized with $\mathrm{AAPH}$ and measurement of the 
remaining fatty acid with GC-FID

Emulsified TAG, such as DDD, EEE, LnLnLn, and OOO, mixed with AAPH was prepared as described in section 2.2. Fifteen screw cap test tubes containing $5 \mathrm{mM}$ LLL emulsion mixed with $1 \mathrm{mM}$ AAPH were covered with aluminum foil and oxidized at $25^{\circ} \mathrm{C}$ for $96 \mathrm{~h}$ in a water bath. Three test tubes were sampled at 0,24 and $48 \mathrm{~h}$ (total of nine test tubes). A same volume of the other emulsified TAG, containing the same concentration of internal standard and AAPH, was added to each of the remaining six test tubes. Three of these test tubes were sampled at $72 \mathrm{~h}$ and the remaining three test tubes were sampled at $96 \mathrm{~h}$. The procedures for the extraction of oxidized TAG, the analysis of the fatty acids, and the evaluation of the unoxidized fatty acids were the same as described in section 2.3.

\subsection{Addition of emulsified TAG into LLL emulsion} previously oxidized with AAPH and measurement of oxygen consumption by biological oxygen monitor

The oxygen consumption of the oxidized TAG emulsion was monitored by a biological oxygen monitor (YSI MODEL 5300 , YSI Incorporation, OH, USA ${ }^{19)}$. Distilled water cooled at $4^{\circ} \mathrm{C}$ was used for the adjustment of the oxygen concentration. Four milliliters of the cooled distilled water was set in the test tube and an oxygen electrode was inserted into the tube from the top with no headspace. The test tube was stirred with a magnetic stirrer at $37^{\circ} \mathrm{C}$ for 30 min and the count monitored on the oxygen monitor display was adjusted to 100 (100 corresponds to $214 \mu \mathrm{M}$ oxygen concentration at $\left.37^{\circ} \mathrm{C}\right)^{20)}$. The TAG emulsion without an internal standard was prepared as indicated in section 2.2. Three milliliters of LLL emulsion was placed in a sample tube and an oxygen electrode was inserted into the tube from the top with no headspace. The tube was set in the oxygen monitoring system and stirred with a magnetic stirrer at $37^{\circ} \mathrm{C}$. Two hundred microliters of $10 \%$ AAPH aqueous solution was added to the stirring emulsion with a microsyringe to initialize oxidation. Emulsified DDD or LLL without internal standard was also prepared by the same method and the concentration was adjusted to $30 \mathrm{mM}$. Five hundred microliters of this emulsion was added to the oxidizing LLL emulsion with a microsyringe when the oxygen count reached 55 (corresponding to an oxygen concentration of $117 \mu \mathrm{M}$ ). Oxygen concentration was continuously plotted on the recorder and the rate of oxygen consumption was calculated from the plotted slope. The oxygen consumption rates before and after the addition of new emulsified TAG were compared.

\subsection{Measurement of LLL and DDD emulsion and their mixture diameters}

The $5 \mathrm{mM}$ LLL and $5 \mathrm{mM}$ DDD emulsions and their mixture were mixed with AAPH aqueous solution and their respective particle diameters were measured with a laser scattering particle size distribution analyzer(LA-300, HORIBA Ltd., Kyoto, Japan) to understand the stability of the emulsions. The mixture of LLL and DDD emulsions was prepared by adding the same volume of each emulsion in the screw cap test tube and mixing the test tube vigorously with a voltex mixer.

2.7 Addition of emulsified DDD into the LLL emulsion previously oxidized with 2,2'-azobisisobutyronitrile (AIBN) and measurement of the remaining fatty acid with GC-FID

LLL was mixed with AIBN and C13 in cyclohexane. Cyclohexane was evaporated under vacuum and an emulsion was prepared using the method described in section 2.2. Final concentrations of LLL, AIBN, and C13 were adjusted to $5 \mathrm{mM}, 1 \mathrm{mM}$, and $10 \mathrm{mM}$, respectively. Fifteen screw cap test tubes containing the LLL emulsion were covered with aluminum foil and oxidized at $25^{\circ} \mathrm{C}$ for $96 \mathrm{~h}$ in a water bath. Three test tubes were sampled at 0, 24 and $48 \mathrm{~h}$ (a total of nine test tubes), and to the same volume of $5 \mathrm{mM}$ DDD emulsion containing the same concentration of internal standard was added. Three of these test tubes were sampled at $72 \mathrm{~h}$ and the remaining three at $96 \mathrm{~h}$. Cyclohexane solution containing $0.5 \%$ butylated hydroxytoluene and $0.05 \%$ triphenylphosphine was added to the test tubes to stop further oxidation and cyclohexane was evaporated with a rotary evaporator under vacuum. The residue in the test tubes was methyl esterified using boron trifluoride methanol solution. The analysis of fatty acids and the evaluation of unoxidized fatty acids were performed as described in section 2.3 .

\section{RESULTS}

\subsection{Oxidation of TAG emulsion with AAPH}

$\beta$-LDL was emulsified and oxidized with the water-soluble radical initiator $\mathrm{AAPH}$ and the relative existence ratio of LA and DHA was analyzed every $24 \mathrm{~h}$ (Fig. 1). The mixture of LLL and DDD (LLL:DDD = 2:1 in molecular ratio) was oxidized and analyzed in the same manner(Fig. 2). DHA oxidized faster than LA in both experiments and about $30 \%$ of the DHA was oxidized. On the contrary, almost none of the LA was oxidized.

\subsection{Effect of emulsified TAG addition on the oxidative rate of LLL emulsion that was previously oxidized with AAPH}

The effect on the oxidation rate of LA by the addition of the DDD emulsion to an LLL emulsion previously oxidized by AAPH is indicated in Fig. 3. The relative amount of LA remaining at respective sampling times was plotted against the initial amount of LA in the emulsion. The addition of DDD could decrease the oxidation rate of LLL. The comparison of the relative amount of LA remaining on average at $96 \mathrm{~h}$ against that at $48 \mathrm{~h}$ is indicated in Fig. 4. The addi- 


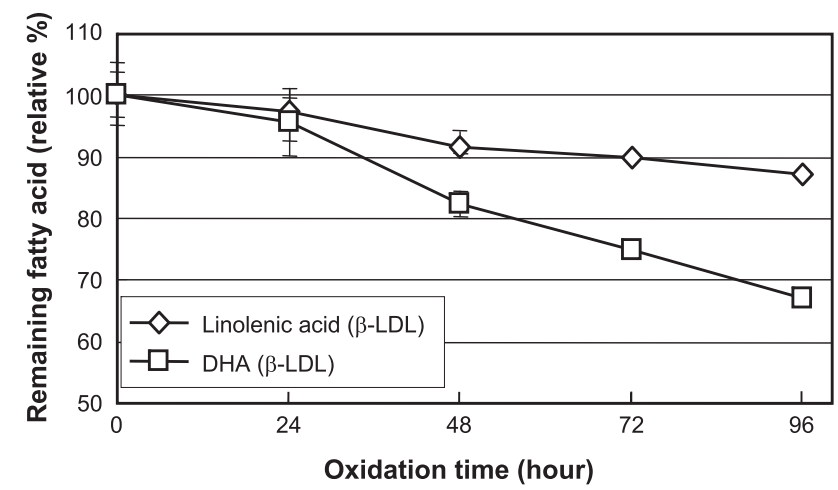

Fig. 1 Changes in relative amount of remaining LA and DHA in emulsified $\beta$-LDL oxidized with watersoluble radical initiator AAPH.

Remaining fatty acid was expressed as a relative existence ratio against the amount at $0 \mathrm{~h}$ and the fatty acid amount at $0 \mathrm{~h}$ was defined as 100 . Each point is indicated in the average of three analyses and standard deviation.

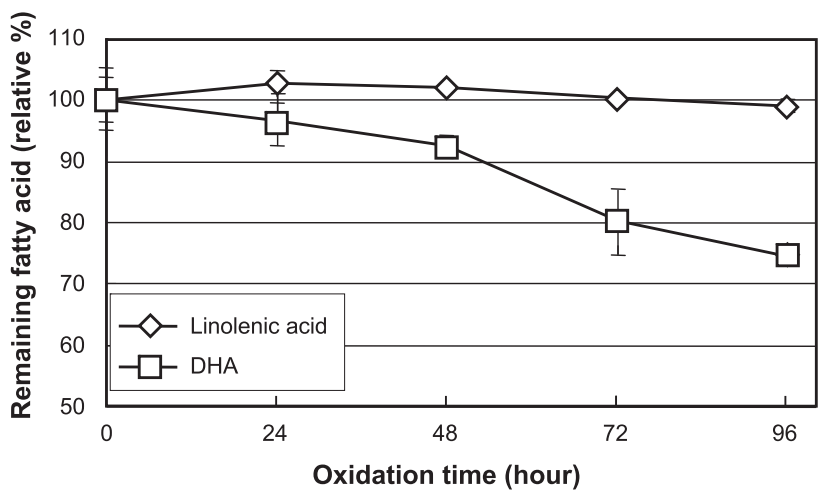

Fig. 2 Changes in relative amount of remaining LA and DHA in emulsified LLL and DDD mixture oxidized with water-soluble radical initiator AAPH.

Expression method is the same as in Fig. 1.

tion of OOO did not change the oxidation amount of LLL drastically as compared to the changes observed after the addition of LnLnLn, EEE, and DDD.

\subsection{Oxygen consumption of emulsified TAG oxidized with $\mathrm{AAPH}$}

Oxygen consumption of the oxidized LLL emulsion before and after the addition of another emulsified TAG was monitored by an oxygen monitor (Fig. 5). The oxidation rate of the LLL emulsion before the addition of a new TAG emulsion was $2.68 \mu \mathrm{M} / \mathrm{min}$. The oxygen concentration increased after the addition of the new TAG emulsion because the new emulsion dissolved a higher concentration of oxygen than did the oxidized LLL. After the addition of

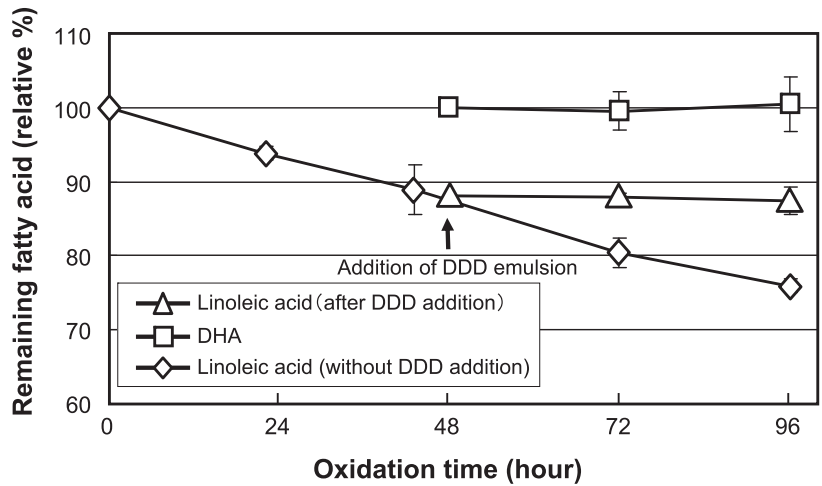

Fig. 3 Changes in relative amount of remaining LA and DHA in emulsified LLL mixed with or without the DDD emulsion at $48 \mathrm{~h}$ oxidized with watersoluble radical initiator AAPH.

Remaining LA was expressed as a relative existence ratio against the amount at $0 \mathrm{~h}$ and the LA amount at $0 \mathrm{~h}$ was defined as 100 . Remaining DHA was expressed as a relative existence ratio against the amount at $48 \mathrm{~h}$ and DHA amount at $48 \mathrm{~h}$ was defined as 100 . Each point is indicated in the average of three analyses and standard deviation.

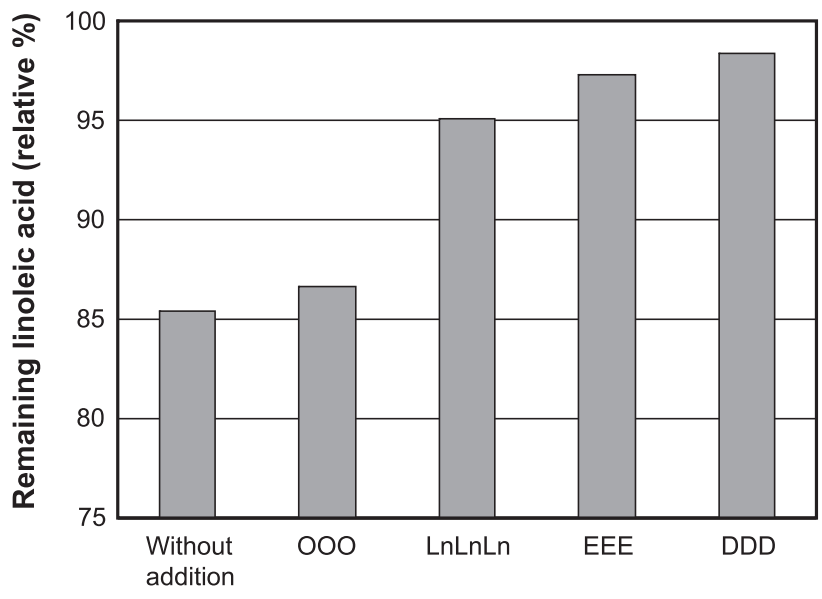

Additional TAG emulsion

Fig. 4 Comparison of the antioxidant ability of four types of emulsions.

Previously oxidized LLL emulsion was mixed with OOO, LnLnLn, EEE, or DDD emulsion at $48 \mathrm{~h}$ after starting oxidation of the LLL emulsion and the relative amount of remaining LA in the LLL emulsion at $96 \mathrm{~h}$ was compared. The values were expressed as a relative ratio of the average amount of LA at $96 \mathrm{~h}$ against that at $48 \mathrm{~h}$.

the DDD emulsion, the oxidation rate of the entire emulsion decreased to $0.53 \mu \mathrm{M} / \mathrm{min}$. In contrast, the addition of 

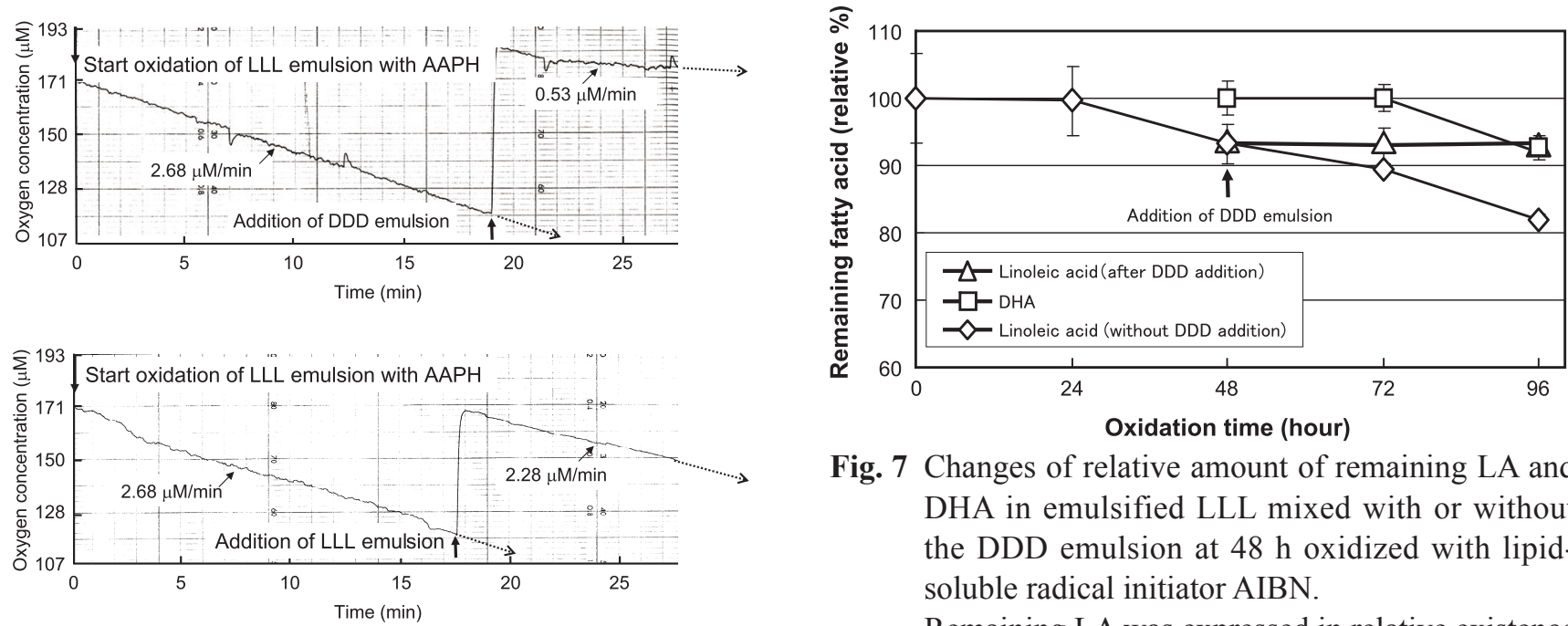

Fig. 5 Comparison of dissolved oxygen consumption rate after addition of DDD or LLL emulsion to oxidizing LLL emulsion.

Increment of dissolved oxygen after addition of fresh emulsion was caused by the dissolved oxygen in the fresh emulsion.

a new LLL emulsion did not change the oxidation rate drastically and the rate of the entire emulsion was $2.28 \mu \mathrm{M} /$ $\min$.

\subsection{Measurement of particle sizes of LLL and DDD emulsions and their mixture}

Particle sizes of the LLL and DDD emulsions and their mixtures are summarized in Fig. 6. The median particle size of all the emulsions was almost $0.5 \mu \mathrm{m}$ and the emulsions were assumed to be stable.
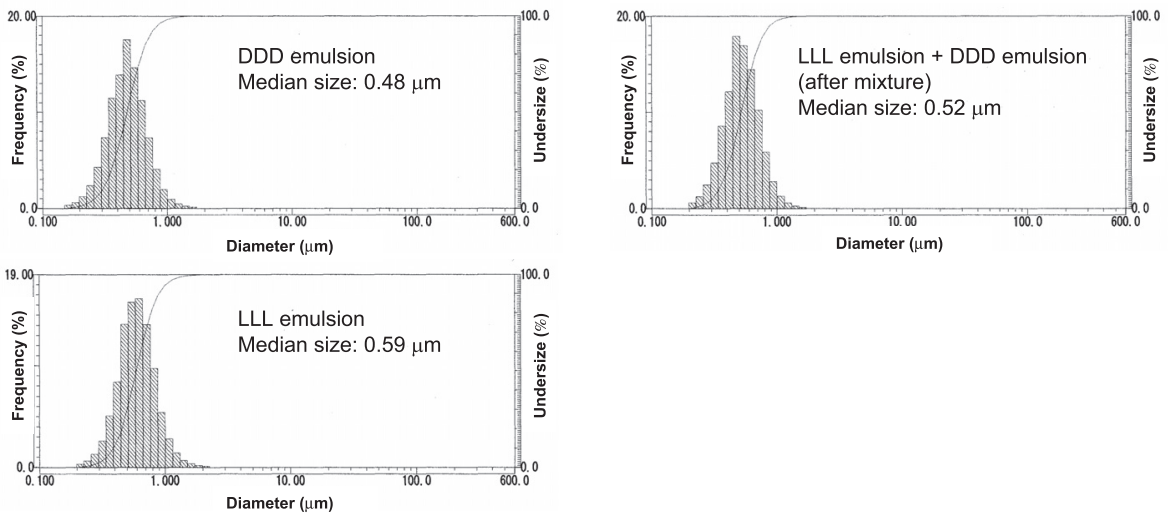

3.5 Effect of emulsified TAG addition on the oxidative rate of LLL emulsion that was previously oxidized with AIBN in emulsion system

The effect on the oxidation rate of LA of adding the DDD emulsion to an LLL emulsion previously oxidized by AIBN is shown in Fig. 7. Addition of DDD also decreased the oxidation rate of LLL. However, the relative percentage of DHA decreased at $96 \mathrm{~h}$.

DHA in emulsified LLL mixed with or without the DDD emulsion at $48 \mathrm{~h}$ oxidized with lipidsoluble radical initiator AIBN.

Remaining LA was expressed in relative existence ratio against the amount at $0 \mathrm{~h}$ and the LA amount at $0 \mathrm{~h}$ was defined as 100 . Remaining DHA was expressed as a relative existence ratio against the amount at $48 \mathrm{~h}$ and the DHA amount at $48 \mathrm{~h}$ was defined as 100. Each point is indicated in an average of three analyses and standard deviation.

Fig. 6 Comparison of particle sizes among LLL, DDD, and mixture of LLL and DDD emulsions. 


\section{DISCUSSION}

Miyashita et al. carried out detailed experiments concerning the characteristic on the oxidation of HUFAs in an emulsified system using many types of fats and oils ${ }^{14)}$, lipid classes $^{11,13,21-25)}$, cell ${ }^{26)}$, emulsifiers ${ }^{27,28)}$, and oxidation systems $^{29)}$. It is recognized that DHA and EPA are highly oxidatively stable fatty acids as compared to LA in emulsified systems, although they are oxidatively unstable fatty acids in bulk system. However, they also compared the stability of DHA and LA in an emulsified DHA and LA mixture and showed that DHA was an oxidizable fatty acid as compared to LA in this mixture system. The same types of experiment, using fatty acid obtained form sardine oil ${ }^{13)}$, supported this finding. Namely, hexaenoic acid (DHA) was also oxidizable as compared to dienoic acid (LA) in micelle consisted of sardine oil fatty acid. This result is inexplicable because emulsified DHA alone was highly oxidatively stable as compared to LA. Miyashita et al. explained this phenomenon that DHA and LA took on more stable conformations against the attack of radicals with increasing molar ratio of DHA to LA, but once DHA in the micelle was oxidized, it behaved like DHA in a bulk system. In our study, we emulsified and oxidized a mixture of DHA and LA with a water-soluble radical initiator, AAPH, but not in the $\mathrm{Fe}^{2+}$ -ascorbic acid system ${ }^{12,22,28)}$ and autoxidation ${ }^{13,14)}$ used by Miyashita et al. The oxidation rate of fatty acid by $\mathrm{Fe}^{2+}$ -ascorbic acid is not constant and depends on the amount of hydroperoxide of the original fatty acids in the emulsion. Therefore, $\mathrm{Fe}^{2+}$-ascorbic acid system is not suitable when a constant oxidation rate is desired. Azo compounds such as water-soluble AAPH and lipid-soluble AIBN can generate peroxy radicals at a constant rate. The half-life of both $\mathrm{AAPH}^{30)}$ and $\mathrm{AIBN}^{31}$ is over $100 \mathrm{~h}$ at $37^{\circ} \mathrm{C}$ and $35^{\circ} \mathrm{C}$, respectively; therefore, it is expected that the peroxy radicals form at a constant rate within $100 \mathrm{~h}$. For these reasons, we used azo compounds in the experiments. The oxidizability of DHA and LA was compared using two types of TAG such as $\beta$-LDL (Fig. 1) and a mixture of DHA and LA (Fig. 2). The tendencies of these two emulsions matched the results in micelle system reported by Miyashita et al. ${ }^{12,13)}$, and DHA could suppress the oxidation of LA located in same or different molecule. However, oxidation of LA was more strongly suppressed by DHA in the mixture of DDD and LLL than in $\beta$-LDL, although both of them consisted of the same ratio of DHA and LA. These results might indicate that an oxidative chain reaction of DHA dominantly occurred in the emulsified mixture system and suppressed the oxidation of LA. The LA in $\beta$-LDL, however, was fixed at the neighborhood of DHA in the structure and the oxidative chain reaction of DHA might have been transmitted to $\mathrm{LA}^{21,25,32)}$. The location of LA in the same molecule of DHA might be a disadvantage from the viewpoint of oxidation of LA. Consequently, DDD and LLL were emulsified separately and their mixture was oxidized by AAPH to sep- arate the location of both the fatty acids. As a result, oxidation of both DHA and LA was strongly suppressed (data not shown). To confirm this phenomenon, emulsified DDD was added at $48 \mathrm{~h}$ to the LLL emulsion oxidized by AAPH(Fig. 3). The oxidation continued for $96 \mathrm{~h}$ and the amount of remaining LA and DHA was quantified. The remaining LA in the emulsified LLL decreased continuously at a constant rate when the DDD emulsion was not added. In contrast, the remaining amount of LA in emulsified LLL was suppressed by the addition of DDD. Furthermore, DHA in emulsified DDD was not oxidized. It was already reported that DHA in an emulsified system is strongly stable against oxidation using several types of lipid classes ${ }^{12,23,24,27)}$. Also, LA mixed with DHA in an emulsified system is stable against oxidation as expressed in Fig. 2. The result of the addition of emulsified DDD to an oxidizing LLL emulsion shows both the merits, and that both of the fatty acids were protected from oxidation. The same experiments were carried out using EEE, LnLnLn, and OOO emulsions and the relative percentage of LA remaining at $96 \mathrm{~h}$ against that at $48 \mathrm{~h}$, namely, the time of addition of another emulsion to the LLL emulsion, was calculated (Fig. 4). Interestingly, increasing the number of double bonds or methylene groups packed by double bonds in the structure increased its ability to suppress the oxidation of LA. In contrast, oleic acid could not suppress the oxidation drastically. These results indicate that the number of double bonds or methylene groups packed by double bonds strongly relates to suppression ability. To understand the ability of the DDD emulsion for the oxidation of LLL emulsion for a short period, the dissolved oxygen consumption rate was measured using a biological oxygen monitor (Fig. 5). As the result, the oxygen consumption rates before and after the addition of the DDD emulsion to an oxidizing LLL emulsion were $2.68 \mu \mathrm{M} / \mathrm{min}$ and $0.53 \mu \mathrm{M} / \mathrm{min}$, respectively. Addition of DDD strongly suppressed the oxidation of not only the LLL but also the DDD emulsion itself, because addition of the LLL emulsion instead of the DDD emulsion did not suppress the oxygen consumption rate (Fig. 5). These results were also observed in the long-period experiment shown in Fig. 3. One possibility (Possibility 1(Fig. 8)) is that two types of emulsions were combined to become one large emulsion by strong mixing. Oxidation behaviors of LA and DHA, however, were completely different from those of the emulsified DDD and LLL mixture because DDD was oxidized when the emulsified mixture of DDD and LLL was oxidized (Fig. 2). Furthermore, the particle size of the mixed LLL emulsion and DDD emulsion was same as that of the LLL or the DDD emulsion(Fig. 6). This result supports the theory that the emulsions did not combine. Another possibility (Possibility 2(Fig. 8) ) is that some kinds of reaction occurred at the surface of each emulsion where two types of emulsified TAGs came in contact. Yazu et al. pointed out the possibility of emulsified HUFA peroxy radi- 

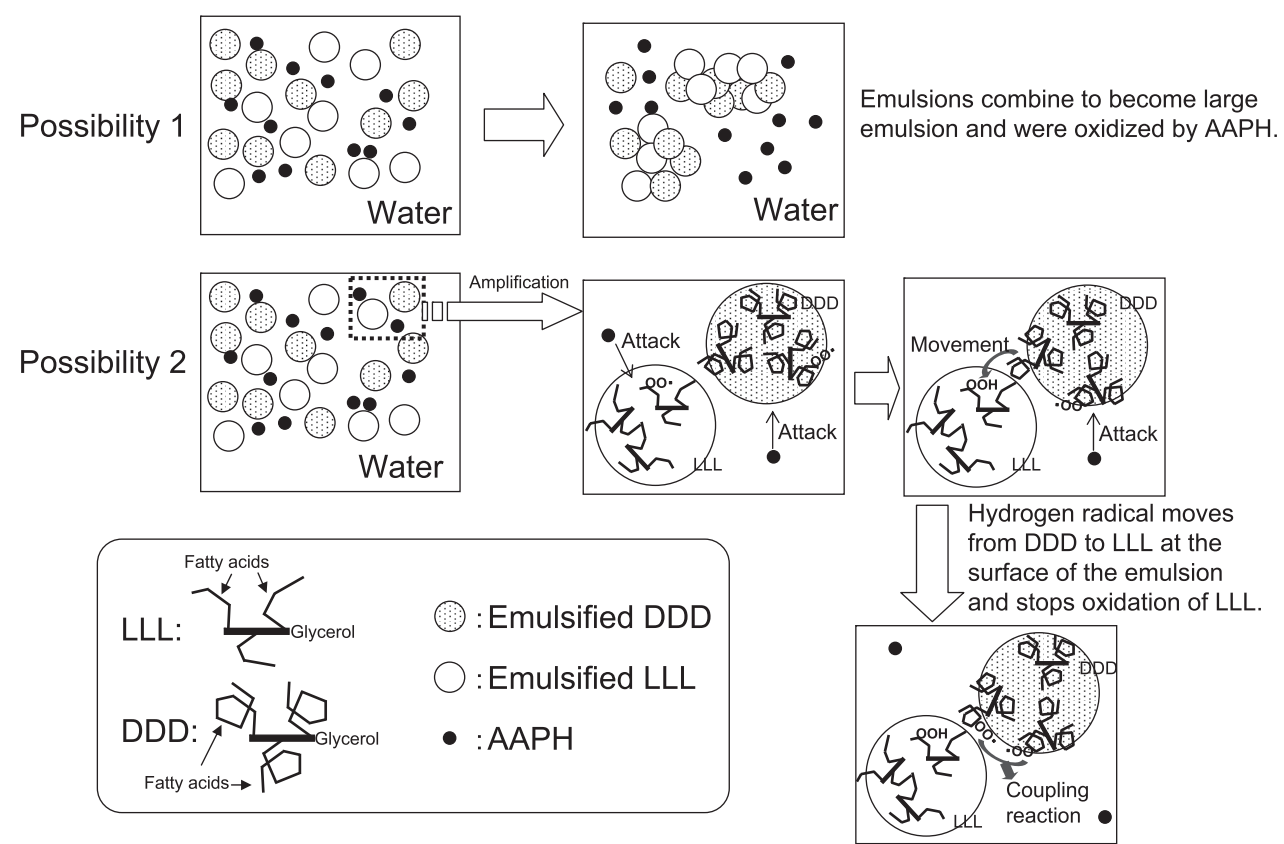

DHA peroxy radical moves to the surface of emulsion and terminates the radical by coupling reaction of between two DHA peroxy radicals or between DHA and AAPH peroxy radicals.

Fig. 8 Hypotheses on oxidation of emulsions in AAPH oxidation system.

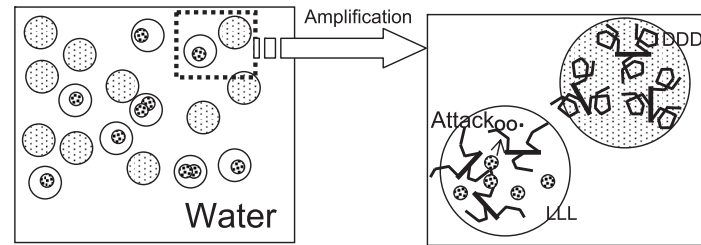

Oxidation starts in the LLL emulsion.
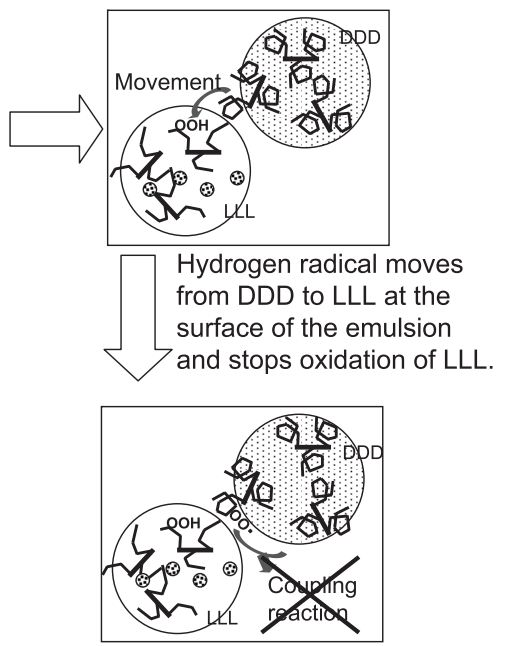

DHA peroxy radical moves to the surface of emulsion but cannot terminates the radical by coupling reaction because almost all radical locate in LLL emulsion. As the result, radical chain reaction occurs in DDD emulsion and DHA was oxidized.

Fig. 9 Hypothesis on oxidation of emulsions in AIBN oxidation system.

cals moving to the surface of the emulsion because of their polarity, and terminate the radical by the coupling reaction of two peroxy radicals ${ }^{15,16)}$, namely the Russel reaction ${ }^{33)}$. According to this idea, the radical formed in the LLL emulsion might move to the surface of the DDD emulsion by the insertion of a DHA peroxy radical into the oxidizing LLL emulsion. The other possibility is that the DDD emulsion captured all the radicals formed in the water phase to terminate the oxidation of LLL because the radical initiator used was water-soluble AAPH. Consequently, the lipid-sol- 
uble radical initiator (AIBN) was employed to oxidize the LLL emulsion to restrict the starting point of oxidation. The half-life of AIBN is over $100 \mathrm{~h}$ at $37^{\circ} \mathrm{C}$; therefore, it is assumed that the peroxy radical was formed at a constant rate during the experimental period at $25^{\circ} \mathrm{C}$. As in the AAPH experiment, the DDD emulsion was added to the LLL emulsion oxidized by AIBN and the consumption of LA and DHA was monitored (Fig. 7). Interestingly, oxidation of the LLL emulsion was also suppressed by the addition of DDD, even in the AIBN oxidation system. This result might indicate that emulsified DHA can insert into and withdraw the radicals from the LLL emulsion. However, remained DHA was decreased at 96 hours in AIBN oxidation system. It may be that a coupling reaction between the peroxy radicals formed from AAPH and DHA occurred easily to terminate any further radical chain reaction of DHA in the AAPH oxidation system because the AAPH peroxy radicals fall to the DDD emulsion constantly. In contrast, the AIBN peroxy radicals are not located in the water phase and it is thought that capturing the DHA peroxy radical, which withdraw radical from oxidizing LLL emulsion, by peroxy radical coupling reaction between AIBN peroxy radical and DHA peroxy radical on the surface of DDD emulsion is difficult. The DHA peroxy radical in the DDD emulsion cannot make the own radical disappear by a coupling reaction to start the radical chain reaction and as a result, DHA decreased at $96 \mathrm{~h}$ (Fig. 9). According to this idea, it is possible to explain the decrease of DHA in the AIBN oxidation system. However, the possibility that AIBN itself might move to the DDD emulsion has not been clarified. Further study is required to understand this phenomenon.

In this study, we indicated that emulsified HUFA might behave as an antioxidant against emulsified LLL in an emulsion system oxidized by azo compounds. In this system, the radical reaction might occur at the surface of the emulsion. However, the condition used in this experiment was special because the oxidation was carried out only by azo compounds and used emulsifier was only TritonX-100. It is reported that the oxidation method and the characteristic of the emulsifier strongly affect the oxidation of emulsified HUFA ${ }^{28)}$. Consequently, further study is required to understand the detailed mechanism of the antioxidant ability of HUFA in an emulsified system.

\section{References}

1) Kamal-Eldin, A. Lipid Oxidation Pathways. The Oil Press. Dundee, Scotland. pp.1-36 (2003).

2) Crampton, E. W.; Common, R. H.; Farmer, F. A.; Berryhill, F. M.; Wiseblatt, L. Studies to determine the nature of the nutritive value of some vegetable oils from heat treatment. II. Investigation of the nutritiousness of the products of thermal polymerization of linseed oil. J. Nutr. 44, 177-189 (1951).

3) Andrews, J. S.; Griffith, W. H.; Wead, J. F. Toxicity of airoxidized soybean oil. J. Nutr. 70, 199-210 (1960).

4) Tovar, L. R. G.; Kaneda, T. Studies on the toxicity of the autoxidized oils IV. Comparative toxicity of secondary oxidation products in autoxidized methyl linoleate. J. Jpn. Oil Chem. Soc. 26, 169-172 (1977).

5) Bragadóttir, M.; Pálmadóttir, H.; Kristbergsson, K. Composition and chemical changes during storage of fish meal from capelin(Mallotus villosus). J. Agric. Food Chem. 52, 1572-1580 (2004).

6) Inagaki, $N$. Regarding food poisoning caused by instant noodle. Food Sanit. Res. Jpn. 16, 370-379 (1966).

7) Gotoh, N.; Watanabe, H.; Osato, R.; Inagaki, K.; Iwasawa, A.; Wada, S. Novel approach on the risk assessment of oxidized fats and oils for perspectives of food safety and quality. I. Oxidized fat and oils induces neurotoxicity relating pica behavior and hypoactivity. Food Chem. Toxicol. 44, 493-498(2006).

8) Natl. Res. Council Nutrient Requirement of Swine. $10^{\text {th }}$ rev. edn. Natl. Acad. Press. Washington D. C. USA. p.126 (1998).

9) De Koning, A. J. The antioxidant ethoxyquin and its analogues: A review. Int. J. Food Prop. 5, 451-461 (2002).

10) Frankel, E. N. Lipid Oxidation. The Oil Press. Dundee, Scotland (1998).

11) Bruna, E.; Petit, E.; Beljean-Leymarie, M.; Huynh, S.; Nouvelot, A. Specific susceptibility of docosahexaenoic acid and eicosapentaenoic acid to peroxidation in aqueous solution. Lipids 24, 970-975 (1989).

12) Miyashita, K.; Nara, E.; Ota, T. Oxidative stability of polyunsaturated fatty acids in an aqueous solution. Biosci. Biotech. Biochem. 57, 1638-1640 (1993).

13) Miyashita, K.; Tateda, N.; Ota, T. Oxidative stability of free fatty acid mixtures from soybean, linseed, and sardine oils in an aqueous solution. Fisheries Sci. 60, 315-318 (1994).

14) Miyashita, K.; Hirao, M.; Nara, E.; Ota, T. Oxidative stability of triglycerides from orbital fat of tuna and soybean oil in an emulsion. Fisheries Sci. 61, 273-275 (1995).

15) Yazu, K.; Yamamoto, Y.; Ukegawa, K.; Etsuo, N. Mechanism of lower oxidizability of eicosapentaenoate than linoleate in aqueous micelles. Lipids 31, 337-340 (1996).

16) Yazu, K.; Yamamoto, Y.; Etsuo, N.; Miki, K.; Ukegawa, K. Mechanism of lower oxidizability of eicosapentaenoate than linoleate in aqueous micelles. II. Effect of antioxidants. Lipids 33, 597-600 (1998).

17) Koga, K.; Ogawa, K.; Nadachi, Y.; Karishu, M; Hatano, K.; Uchida, Y. Process for producing DHA-containing tofu or soybean milk drink, or dry powder thereof. 
USP5976606.

18) Gotoh, N.; Moroda, K.; Watanabe, H.; Yoshinaga, K.; Tanaka, M.; Mizobe, H.; Ichioka, K.; Tokairin, S; Wada, $\mathrm{S}$. Metabolism of odd-numbered fatty acids and evennumbered fatty acids in mouse. J. Oleo Sci. 57, 293-299 (2008).

19) Gotoh, N.; Shimizu, K.; Komuro, E.; Tsuchiya, J.; Noguchi, N.; Niki, E. Antioxidant activities of probucol against lipid peroxidations. Biochem. Biophys. Acta 1128, 147-154(1992).

20) http://antoine.frostburg.edu/chem/senese/101/solu tions/faq/predicting-DO.shtml\#standardmethods (as of 11th May 2010).

21) Miyashita, K.; Takagi, T. Autoxidation rates of various esters of safflower oil and linoleic acid. J. Am. Oil Chem. Soc. 65, 1156-1158(1988).

22) Hirano, S.; Miyashita, K.; Ota, T.; Nishikawa, M.; Maruyama, K.; Nakayama, S. Aquous oxidation of ethyl linoleate, ethyl linolenate, and ethyl docosahexaenoate. Biosci. Biotech. Biochem. 61, 281-285 (1997).

23) Nara, E.; Miyashita, K.; Ota, T. Oxidative stability of $\mathrm{PC}$ containing linoleate and docosahexaenoate in aqueous solution with or without chicken egg albumin. Biosci. Biotech. Biochem. 59, 2319-2320 (1995).

24) Nara, E.; Miyashita, K.; Ota, T. Oxidative stability of liposomes prepared from soybean PC, chicken egg PC, and salmon egg PC. Biosci. Biotech. Biochem. 61, 1736-1738 (1997)

25) Miyashita, K.; Hirano, S.; Itabashi, Y.; Ota, T.; Nishikawa, M.; Nakayama, S. Oxidative stability of polyunsaturated monoacylglycerol and triacylglycerol in aqueous micelles. J. Jpn. Oil Chem. Soc. 46, 205-208(1997).

26) Araseki, M.; Kobayashi, H.; Hosokawa, M.; Miyashita, K.
Lipid peroxidation of a human hepatoma cell line (HepG2) after incorporation of linoleic acid, arachidonic acid, and docosahexaenoic acid. Biosci. Biotech. Biochem. 69, 483-490(2005).

27) Miyashita, K.; Inukai, N.; Ota, T. Effect of Tween 20 on the oxidative stability of sodium linoleate and sodium docosahexaenoate. Biosci. Biotech. Biochem. 61, 716-717 (1997).

28) Miyashita, K.; Nara, E.; Ota, T. Comparative study on the oxidative stability of phosphatidylcholines from salmon egg and soybean in aqueous solution. Biosci. Biotech. Biochem. 58, 1772-1775 (1994).

29) Araseki, M.; Yamamoto, K.; Miyashita, K. Oxidative stability of polyunsaturated fatty acid in phosphatidylcholine liposomes. Biosci. Biotech. Biochem. 66, 2573-2577(2002).

30) Yoshida, Y.; Itoh, N.; Saito, Y.; Hayakawa, M.; Niki, E. Application of water-soluble radical initiator, 2,2' -azobis-[2-(2-imidazolin-2-yl) propane] dihydrochoride, to a study of oxidative stress. Free Radical Res. 38, 375-384 (2004).

31) Li, H.; Han, B. Impregnation of polyethylene (PE) with styrene using supercritical $\mathrm{CO}_{2}$ as the swelling agent and preparation of $\mathrm{PE} /$ polystyrene composites. Ind. Eng. Chem. Res. 39, 4506-4509(2000).

32) Endo, Y.; Hoshizaki, S.; Fujimoto, K. Oxidation of synthetic triacylglycerols containing eicosapentaenoic and docosahexaenoic acids: Effect of oxidation system and triacylglycerol structure. J. Am. Oil Chem. Soc. 74, 1041-1045(1997).

33) Russell, G.A. The competitive oxidation of cumene and tetralin. J. Am. Chem. Soc. 77, 4583-4590 (1955). 